\title{
On-Chip Microwave Quantum Hall Circulator
}

\author{
A. C. Mahoney, ${ }^{1,2}$ J. I. Colless, ${ }^{1,2, *}$ S. J. Pauka, ${ }^{1,2}$ J. M. Hornibrook, ${ }^{1,2}$ J. D. Watson, ${ }^{3,4}$ G. C. Gardner, ${ }^{4,5}$ \\ M. J. Manfra, ${ }^{3,4}$ A. C. Doherty, ${ }^{1}$ and D. J. Reilly ${ }^{1,2, \dagger}$ \\ ${ }^{1}$ ARC Centre of Excellence for Engineered Quantum Systems, School of Physics, The University of Sydney, \\ Sydney, New South Wales 2006, Australia \\ ${ }^{2}$ Station Q Sydney, The University of Sydney, Sydney, New South Wales 2006, Australia \\ ${ }^{3}$ Department of Physics and Astronomy, Purdue University, West Lafayette, Indiana 47907, USA \\ ${ }^{4}$ Birck Nanotechnology Center, School of Materials Engineering and School of Electrical \\ and Computer Engineering, Purdue University, West Lafayette, Indiana 47907, USA \\ ${ }^{5}$ Station Q Purdue, Purdue University, West Lafayette, Indiana 47907, USA \\ (Received 10 September 2016; revised manuscript received 15 November 2016; published 24 January 2017)
}

\begin{abstract}
Circulators are nonreciprocal circuit elements that are integral to technologies including radar systems, microwave communication transceivers, and the readout of quantum information devices. Their nonreciprocity arises from the interference of microwaves over the centimeter scale of the signal wavelength, in the presence of bulky magnetic media that breaks time-reversal symmetry. Here, we realize a completely passive on-chip microwave circulator with size $1 / 1000$ th the wavelength by exploiting the chiral, "slow-light" response of a two-dimensional electron gas in the quantum Hall regime. For an integrated GaAs device with $330 \mu \mathrm{m}$ diameter and about 1-GHz center frequency, a nonreciprocity of $25 \mathrm{~dB}$ is observed over a 50-MHz bandwidth. Furthermore, the nonreciprocity can be dynamically tuned by varying the voltage at the port, an aspect that may enable reconfigurable passive routing of microwave signals on chip.
\end{abstract}

DOI: 10.1103/PhysRevX.7.011007

Subject Areas: Condensed Matter Physics, Mesoscopics

\section{INTRODUCTION}

Miniaturized, nonreciprocal devices are currently of broad interest for enabling new applications in acoustics [1], photonics [2,3], transceiver technology [4], and in the regime of near quantum-limited measurement [5-9], where they are needed to isolate qubits from their noisy readout circuits. Since the 1950s, passive circuit elements exhibiting nonreciprocity at microwave frequencies have been implemented using bulky magnetic devices that are comparable in scale to the centimeter wavelength of signals in their operating band. The footprint of these components poses a major limitation to integrating entire systems on a chip, such as what is required, for instance, to scale up quantum computing technology.

A seemingly obvious means of realizing nonreciprocity on a semiconductor chip is to use the Hall effect, where an external magnetic field breaks the time-reversal symmetry of electrical transport [10]. Hall-based devices were ruled out in 1954, however [11], since near the electrical contacts,

\footnotetext{
* Present address: Department of Physics, University of California, Berkeley, CA 94720, USA.

${ }^{\dagger}$ To whom correspondence should be addressed. david.reilly@sydney.edu.au

Published by the American Physical Society under the terms of the Creative Commons Attribution 4.0 International license. Further distribution of this work must maintain attribution to the author(s) and the published article's title, journal citation, and DOI.
}

where the current and voltage are collinear, dissipation is so significant that the usefulness of this approach is greatly limited. This dissipative mechanism has an analog in the quantum Hall regime where the two-terminal resistance of a device is always finite over a scale of the inelastic scattering length as carriers transition from their contacts to the dissipationless, one-dimensional (1D) edge states that support transport [12]. Recently, Viola and DiVincenzo [9] proposed a means of addressing the limitation imposed by two-terminal dissipation, suggesting the possibility of coupling microwave signals to the edge of a quantum Hall device reactively, without using traditional ohmic contacts. In a geometry where the signal ports of the device are positioned orthogonal to an incompressible quantum Hall edge state, microwave power is coupled capacitively, and nondissipative transport in one direction appears possible [9].

Here, we engineer, on chip, a chiral microwave interferometer that yields a high degree of nonreciprocity and dynamic range, with the low-dissipation inherent to edge transport in the quantum Hall regime. Configured as a completely passive three-port circulator, our device demonstrates nonreciprocal operation at frequencies and magnetic fields commonly used for the readout of spin qubits [13-16], facilitating integration with such semiconductor technologies. In comparison to traditional ferrite-based microwave components, this quantum Hall circulator is reduced in size by a factor of about $1 / 1000$ th the wavelength and exhibits a new mode of operation in which circulation can be dynamically reconfigured either by applying a dc voltage to the port electrodes or by altering 
the strength of the magnetic field. A simple model based on a Fano-resonance mechanism [17] qualitatively accounts for the observed phenomena.

\section{EXPERIMENTAL SETUP AND RESULTS}

\section{A. Transmission-line spectroscopy of EMP modes}

Central to the operation of our device are edge magnetoplasmons (EMPs), resonant modes of the electron gas first observed in the classical response of electrons on the surface of liquid helium $[18,19]$. Such excitations have since been found to propagate along a quantum Hall edge in response to a capacitively coupled microwave excitation [20-27]. These chiral excitations travel with a velocity $v_{\text {EMP }} \sim|\vec{E}| /|\vec{B}|$, set by the ratio of the electric field $\vec{E}$ at the sample boundary and the applied magnetic field $\vec{B}[22]$.
For a high-mobility two-dimensional electron gas (2DEG) formed at the interface of the semiconductors GaAs and AlGaAs (see Ref. [28] for details), the velocity of the EMP modes is typically $v_{\mathrm{EMP}} \sim 10^{5} \mathrm{~ms}^{-1}[29,30]$, some 1000 times slower than the speed of light in the semiconductor dielectric. In order to exploit these EMPs to realize nonreciprocal microwave devices, we first detect their presence in a contactless etched disk of quantum Hall fluid by coupling to a proximal metallic coplanar transmission line (CTL) [31], as shown in Figs. 1(a) and 1(b). By measuring the transmitted microwave power through the CTL as a function of frequency $f$, a spectrum of discrete features is observed with applied magnetic field $B$ [Fig. 1(c)]. We identify EMP modes in the data with frequencies set by the edge velocity and circumference of the disk, following the dependence $f \sim$ $B^{-1}\left[\log \left(B^{2}\right)+\right.$ const $]$ [20], and extract the effective dielectric constant $\epsilon^{*} \approx 8.7$, consistent with the propagation (a)

(b)

(a)

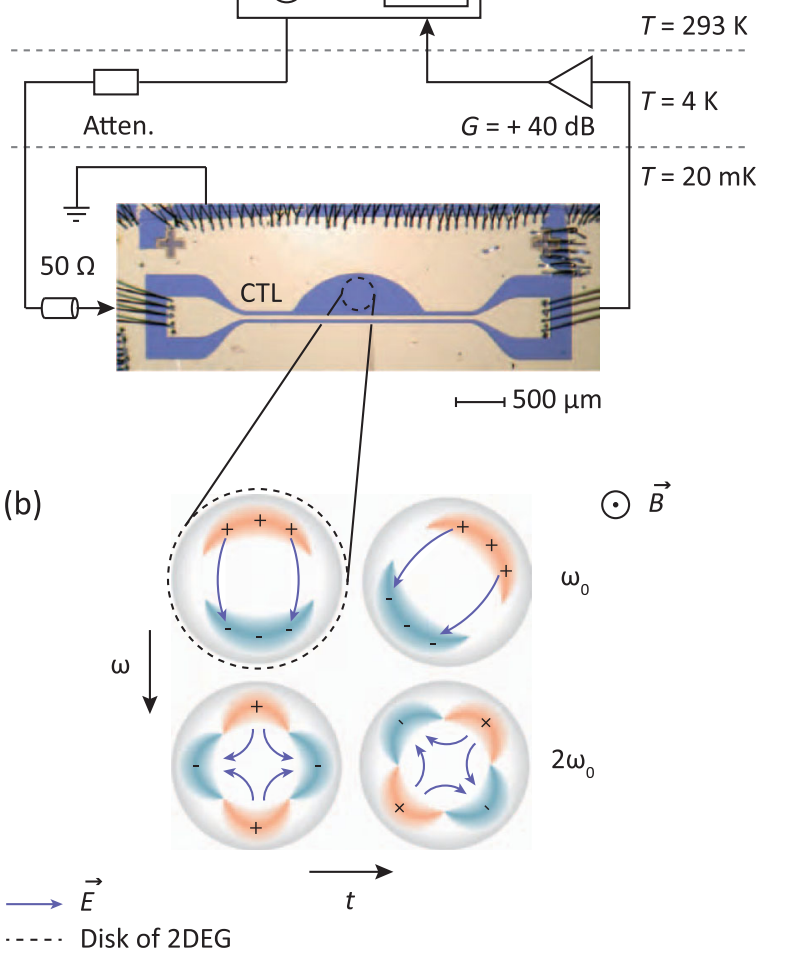

(c) Absorption $\Delta \mathrm{S} 21(\mathrm{~dB})$
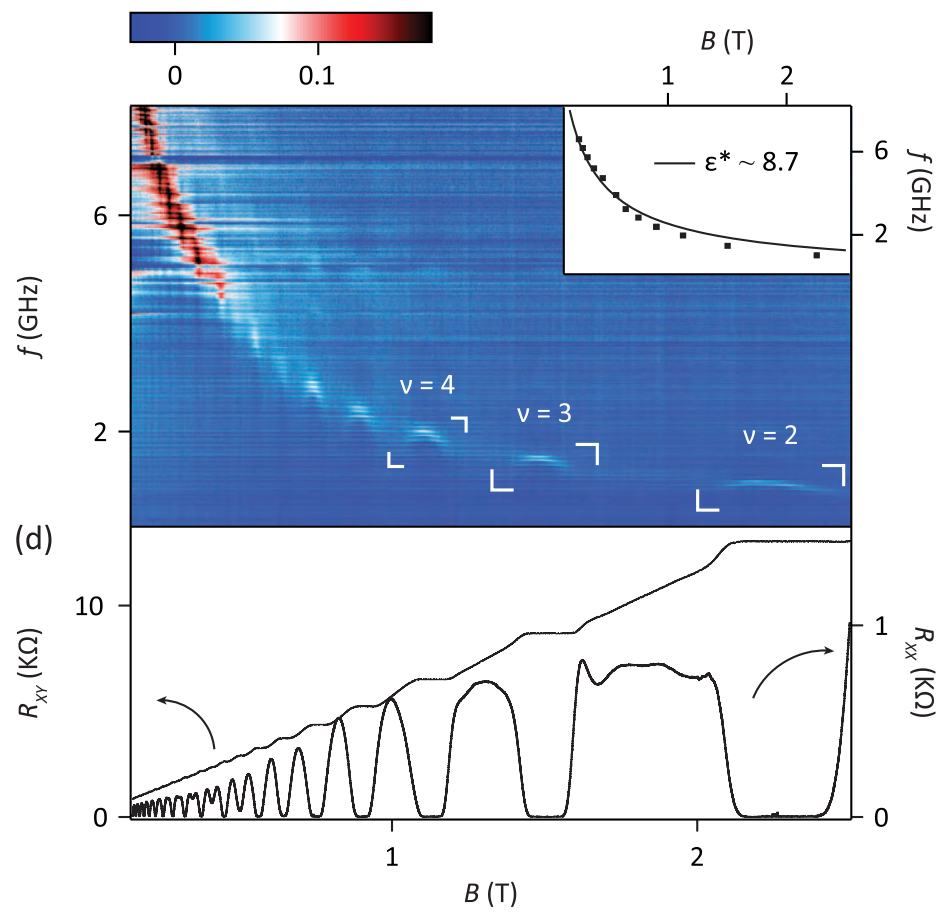

FIG. 1. Detecting microwave edge magnetoplasmons. (a) Experimental setup including photograph of a coplanar transmission-line device similar to that used to perform measurements coupled to a 350- $\mu$ m etched disc of 2DEG (black dashed circle) at a fridge temperature of $T=20 \mathrm{mK}$. A vector network analyzer is used to excite EMP modes across a wide frequency range, and microwave absorption is measured as the ratio of the amplified output to input signal $\left(S_{21}\right)$ from the CTL. (b) Illustration of the fundamental (top row) and first harmonic (bottom row) EMP modes as they evolve with time, where $\omega_{0}$ is the fundamental mode and $2 \omega_{0}$ the first harmonic (adapted from Ref. [20]). Charge distributions and electric fields $\vec{E}$ are indicated schematically. An external magnetic field $B$ applied to the device points out of the page. (c) EMP spectrum of the quantum Hall disk showing absorbed microwave power as a function of frequency and magnetic field. A background obtained at high field has been subtracted from the data. The inset shows the position of absorption dips at integer quantum Hall filling factors. The black line is a fit that allows an average effective dielectric constant of $\epsilon^{*} \approx 8.7$ to be extracted, consistent with excitations of an edge state in GaAs (see Ref. [28]). (d) Transverse ( $\left.R_{x y}\right)$ and longitudinal $\left(R_{x x}\right)$ Hall resistance measurements taken at $T=20 \mathrm{mK}$ on a Hall bar proximal to the microwave disk. The $2 \mathrm{DEG}$ is $270 \mathrm{~nm}$ below the surface with carrier density $\mathrm{n}_{s}=1.1 \times 10^{11} \mathrm{~cm}^{-2}$ and mobility $\mu=5.2 \times 10^{6} \mathrm{~cm}^{2} / \mathrm{Vs}$. 
medium [23,32] (see Ref. [28]). Comparing the microwave spectrum to transport measurements from a Hall bar on the same chip [Fig. 1(d)], we note that at high fields (with only the last few Landau levels occupied), features resolve into discrete, crescent-shaped resonances that coincide with minima in the longitudinal resistance $R_{x x}$, where dissipation is suppressed.

\section{B. Three-port circulator}

To test if these edge magnetoplasmons support the nonreciprocal transmission of microwaves, we implement a standard circulator configuration, with three ports arranged at 120-degree intervals around a disk of $2 \mathrm{DEG}$ (330 $\mu \mathrm{m}$ diameter), as shown in Figs. 2(a) and 2(b). For a single edge at a high magnetic field, a voltage applied to a port capacitance induces an orthogonal current in the edge state, with an impedance of the order of the inverse conductance quantum (about $26 \mathrm{k} \Omega$ ). Given that our present measurement setup uses electronic components with a characteristic impedance of $Z_{0} \sim 50 \Omega$, we have added an impedance-matching circuit to enhance the response of each port. This network comprises a series chip-inductor $L=47 \mathrm{nH}$ in resonance with the stray capacitance $C_{\text {stray }}$. The impedance of the Hall edge could be lowered closer to $Z_{0}$ by connecting multiple 2DEG circulators in parallel [33] or by taking advantage of recently proposed "self-matching" port configurations [34,35] (see Ref. [28] for detailed discussion). The circulator is also embedded in a reflectometry arrangement [Fig. 2(c)] that enables a measurement of the port reflection as well as the port transmission coefficient, from which dissipation can be estimated. As a control, we first measure all microwave $S$ parameters at zero magnetic field, observing that all directions and ports are equivalent, as shown in Fig. 2(d). An overall frequency-dependent but reciprocal response can be associated with the impedance matching network, with matching frequency set to $1 / \sqrt{L C_{\text {stray }}} \sim 1 \mathrm{GHz}$. All subsequent measurements are normalized relative to this zero-field transmission response.

Turning to our key result, Fig. 3 shows the full transmission response of the three-port circulator in the presence of a magnetic field that breaks time-reversal symmetry. Similar to the EMP spectrum of Fig. 1(c), we first observe the presence of EMPs which enhance the transmitted power at certain frequencies, broadly following an approximate $f \sim B^{-1}$ dispersion relation, as is seen in Fig. 3(a) $\left(S_{13}\right)$ and Fig. 3(b) $\left(S_{31}\right)$. Strikingly, there are regions of the spectrum where the transmitted power appears to flow in either a forward or reverse direction with respect to the chirality of the edge. Particularly apparent are the crescent-shaped features that switch from forward to reverse transmission at distinct frequencies. This phenomenon, with a peak near the fundamental frequency of the EMP mode and a dip near the first EMP harmonic, is seen for all $S$ parameters in the
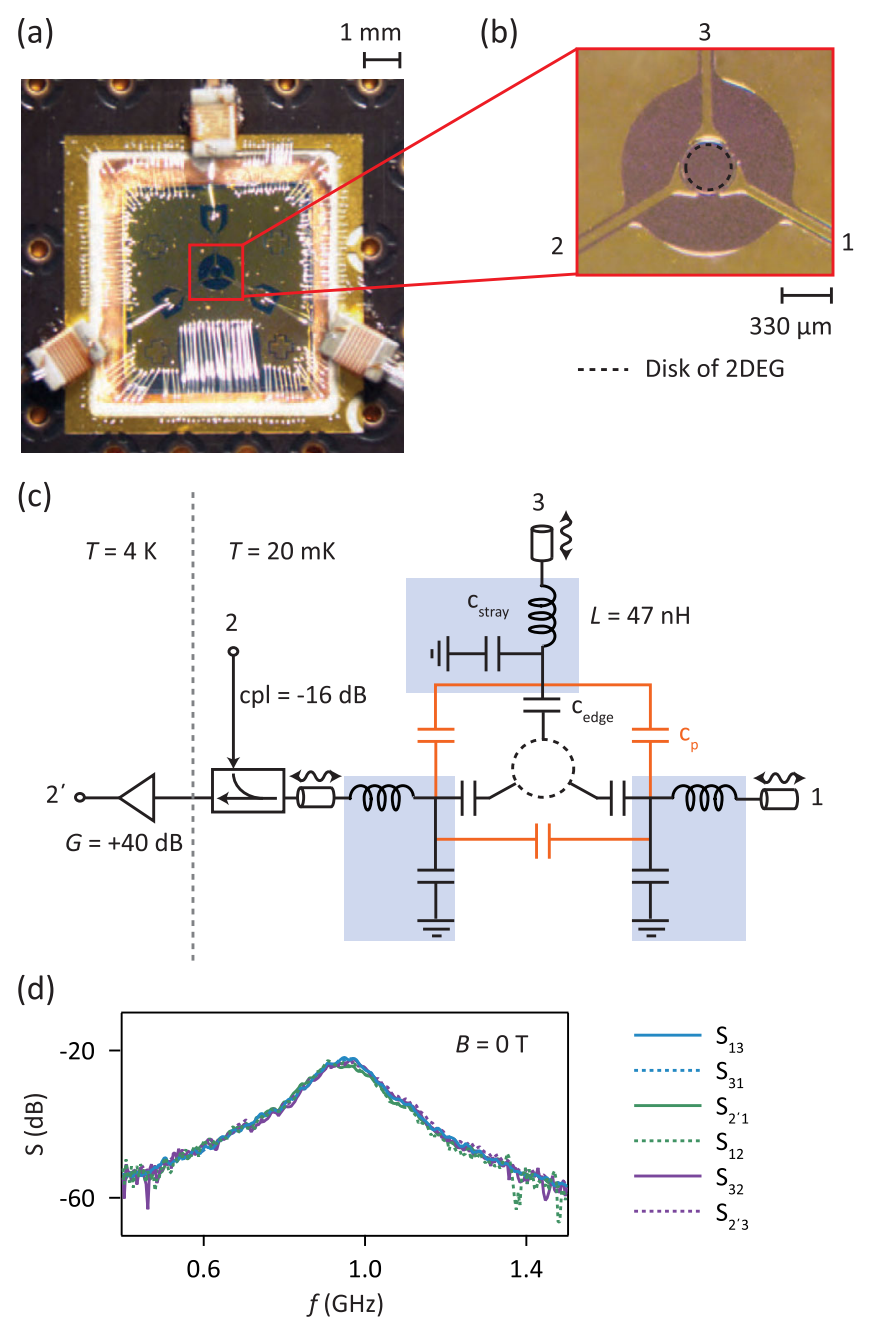

FIG. 2. Experimental setup for determining the response of the on-chip circulator. (a) Photograph of circulator device showing the three coplanar transmission lines connected to copper wirewound chip inductors for impedance matching. (b) Close-up of false-colored photo of the circulator showing a 330- $\mu \mathrm{m}$ diameter 2DEG disc with a $20-\mu \mathrm{m}$ gap to the metal defining the three signal ports. (c) Circuit schematic of the experimental setup indicating port-to-edge capacitive coupling $C_{\text {edge }}$ and direct parasitic coupling between ports $C_{p}$. Resonant ( $L C_{\text {stray }}$ ) matching circuits are indicated with blue boxes. The input of port 2 passes through a directional coupler, with the reflected signal coupled to the output line (denoted $2^{\prime}$ ) and amplified at $4 \mathrm{~K}$. (d) Full six-way transmission response of the circulator at zero magnetic field, with $S$-parameter measurements indicating complete reciprocity and a frequency response that arises from the matching networks. For each port, the measured response of the amplifiers, couplers, and cold attenuators in the circuit has been subtracted.

chiral (clockwise) direction of the three-port device [see solid lines in Fig. 3(d)].

To measure the extent of nonreciprocity in our circulator, Fig. 3(c) shows the difference between forward and reverse power by subtracting $S_{31}$ from $S_{13}$. Unlike the $B=0$ data shown in Fig. 2(d), we now observe a strong directional 
(a)

(b)

(c)

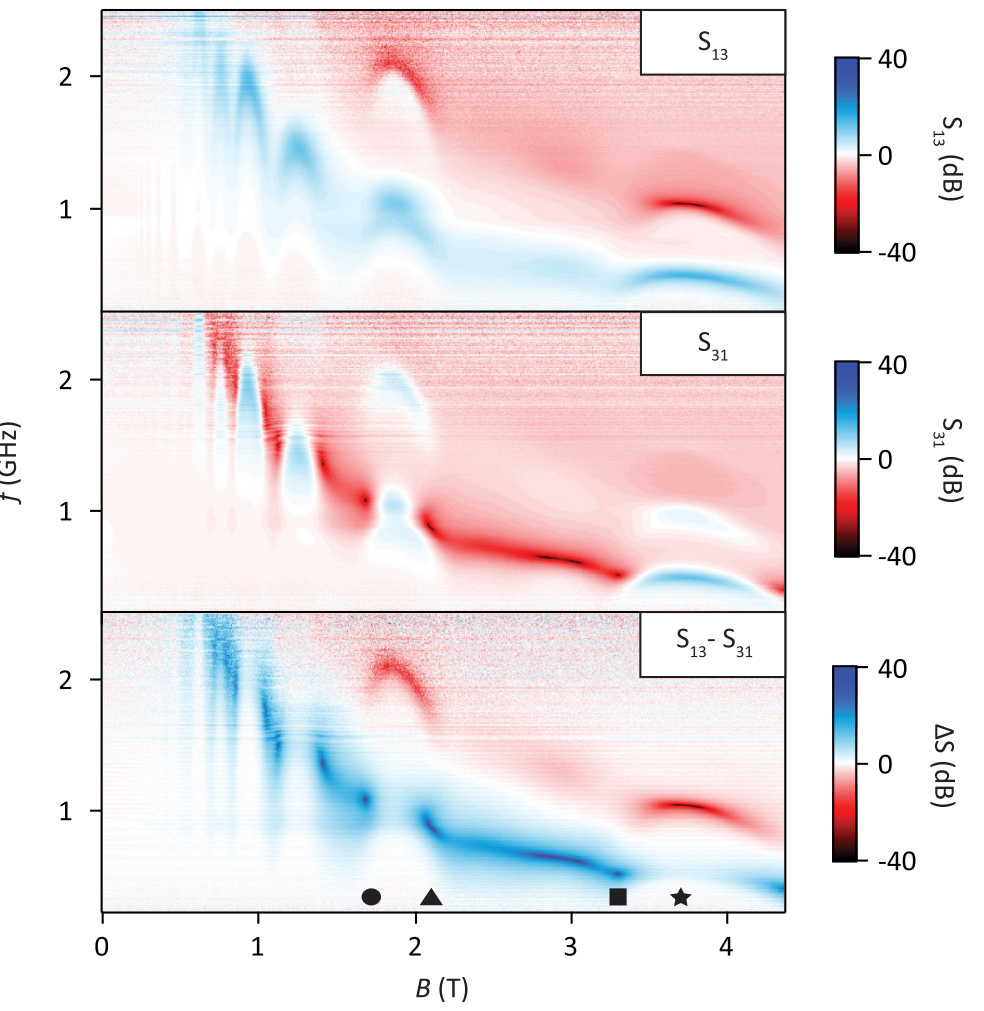

(d)

(e)

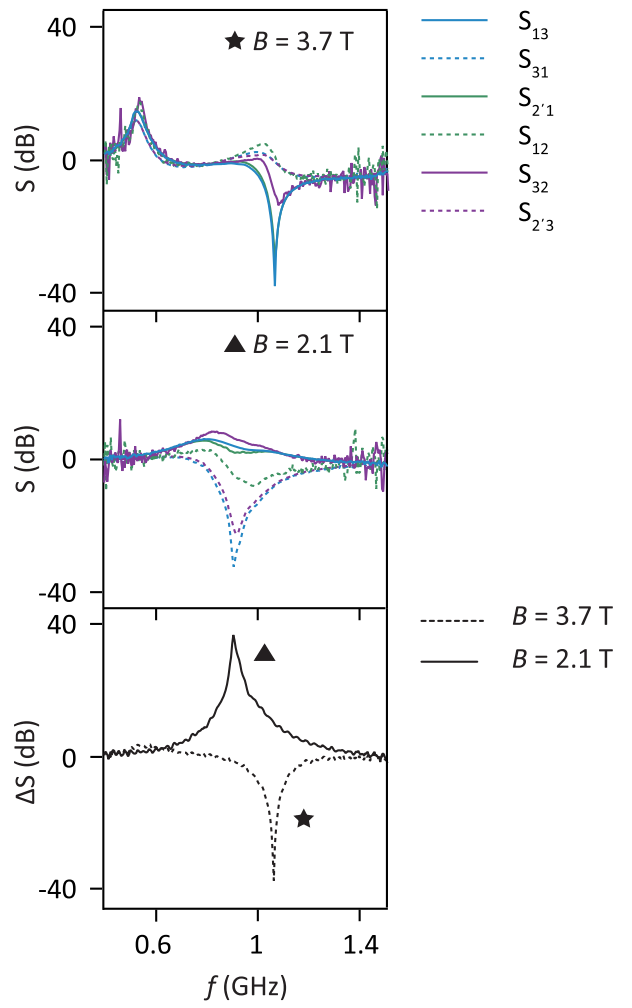

(g)

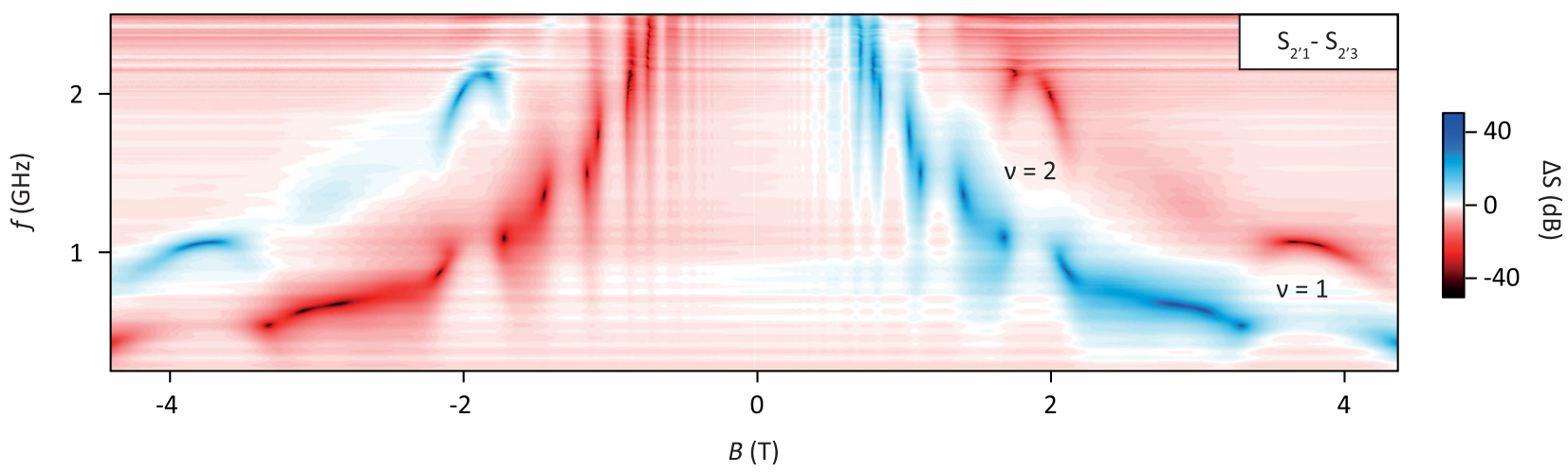

FIG. 3. Nonreciprocal response of the quantum Hall circulator. (a,b) Port transmission $S_{13}$ and $S_{31}$ with frequency and magnetic field. All measurements have been normalized to the gain-corrected background at $B=0$ [shown in Fig. 2(d)], which defines the $0 \mathrm{~dB}$ point on the color scale. (c) Microwave response $S_{13}-S_{31}$ showing strong frequency and $B$-dependent nonreciprocity. (d,e) Full combination of transmission $S$ parameters, taken at $B$ fields indicated by the symbols in (c). (f) Cuts through the color scale data in (c), demonstrating forward and reverse circulation. (g) Isolation, $\Delta S=S_{2^{\prime} 1}-S_{2^{\prime} 3}$, measured at positive and negative magnetic fields. Note that the positions of features are symmetric about the $B=0$ axis, but with opposite sign $\Delta S$.

dependence in the isolation between ports that approach $40 \mathrm{~dB}$ at particular frequencies and magnetic fields [Fig. 3(f)]. Alternatively, we can also test for nonreciprocity by comparing the response of signals from two different inputs of the circulator to a common output. Since the device is geometrically symmetric, the responses from the separate paths $S_{2^{\prime} 1}$ and $S_{2^{\prime} 3}$ are the same at $B=0$ [see Fig. 2(d)]. In the presence of a magnetic field, however, Fig. 3(g) shows that these paths are no longer equivalent but, rather, depend on the direction of the field. This is evident in the data since blue and red features are not mirrored about $B=0$.
Comparing the microwave response of the circulator to independent quantum Hall transport data suggests two distinct regimes. Between integer filling factors, where $R_{x x}$ is maximized in transport, there is a large nonreciprocity in the microwave response, but there is also likely strong dissipation. Contrasting these broad regions are narrow crescent-shaped features that occur at fields corresponding to integer filling. These narrow features are particularly strong at frequencies near twice the fundamental EMP resonance. Again, overlaying these features with transport measurements indicates that they align with minima in $R_{x x}$, where dissipation is suppressed. A direct 
and accurate measurement of the microwave dissipation is challenging in the regime where the impedance of the device is mismatched. Nevertheless, by accounting for the transmitted and reflected signal power, we find the dissipation to be a few percent, consistent with the value of about $1 \%$ given by our model (discussed below).

\section{DISCUSSION AND MODEL}

We account for the distinct features in our measurements, as well as the phenomena of forward and reverse circulation via a simple picture of a Fano-like resonance. Figure 4 illustrates the phenomenology of the quantum Hall circulator. Similar to the operation of a traditional ferrite device, we consider a resonator structure with two interfering paths, as shown in Fig. 4(a). The arms of this interferometer comprise a direct path, supported by the parasitic
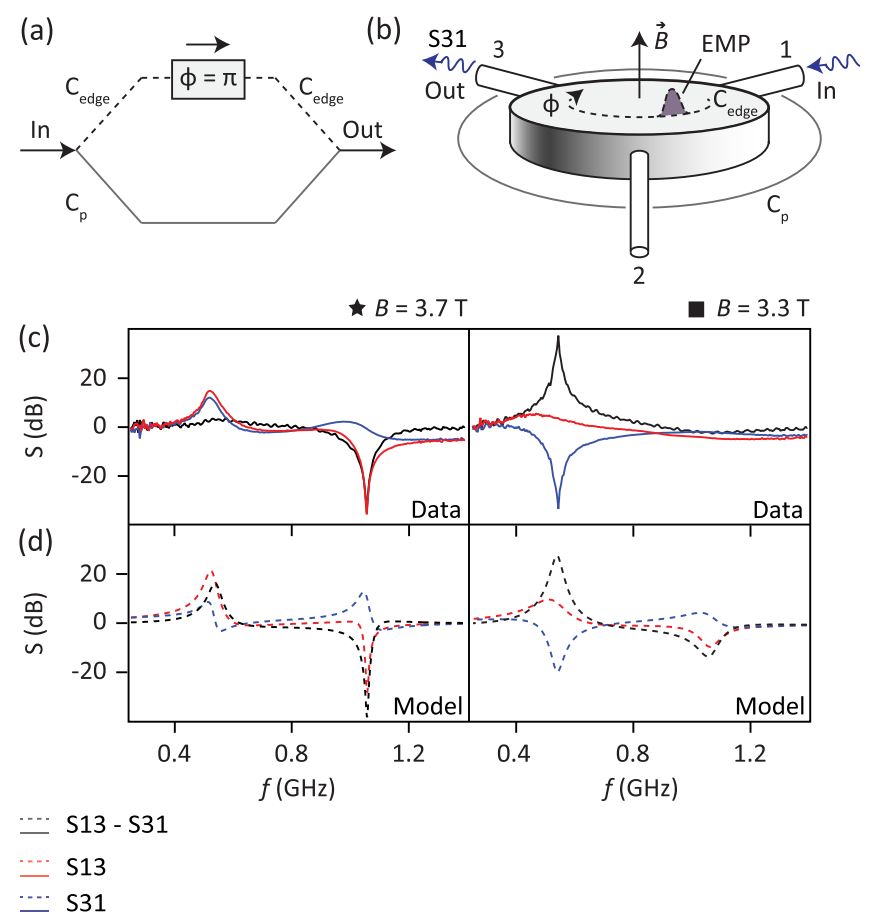

FIG. 4. Comparison of data and model. (a,b) Proposed interferometric mechanism underlying the operation of the quantum Hall circulator, with a slow plasmonic path, via $C_{\text {edge }}$, and a direct capacitive path, via $C_{p}$. A nonreciprocal response between ports is produced for frequencies where the two paths are out of phase by $\phi=\pi$ in the forward direction and $\phi=2 \pi$ in the reverse direction. (c,d) Comparison of a simple model that captures this physics (bottom graphs), with experimental data (top graphs), at two different magnetic fields indicated by the symbols [with respect to Fig. 3(c)]. The model is described in detail in the Appendix, with parameters set to values $Z_{0}=50 \Omega$, $C_{p}=315 \mathrm{fF}, C_{\text {edge }}=127 \mathrm{fF}, R_{x y}=5000 \Omega$, with $R=80 \Omega$ in the center of an EMP resonance (star symbol) and $R=350 \Omega$ off resonance (square symbol). Note that the impedancematching network transforms $R_{x y}=25 \mathrm{k} \Omega$ towards a few $\mathrm{k} \Omega$, consistent with the value used in the model. (geometric) capacitance $C_{p}$ between ports, and an indirect path $C_{\text {edge, }}$ which capacitively couples ports via the plasmonic excitation of a quantum Hall edge. Key to the operation of our circulator is this "slow light" response of the EMP modes, which, traveling at velocities 1000 times slower than the microwaves in the direct path, acquire the same phase over a length scale that is 1000 times shorter than the microwave wavelength in the dielectric. Considering these two paths, we note that there will be a frequency near the EMP resonance, at which the phase acquired via the edge leads to complete destructive interference with the signal propagating via the direct path. Given the chirality of the EMP, the condition for destructive interference will be dependent on the direction of microwave transmission, producing a nonreciprocal response between adjacent ports. Take, for instance, the case where signals from port 3 to 1 propagate clockwise via the edge capacitance $C_{\text {edge }}$ and acquire a phase of $\pi$ radians with respect to the signal traveling via $C_{p}$. Interference of these signals isolates port 1 , whereas reverse transport, from port 1 back to port 3 , must continue in a clockwise direction, past port 2 , and acquire a constructive phase of $2 \pi$ over twice the length. Circulation in the opposite direction to the chirality of the edge can now be understood for frequencies in which a $\pi$ phase is acquired in the forward direction, but a $2 \pi$ phase is acquired in reverse.

We construct a simple model based on this Fano-like picture of interfering paths [17], by modifying the standard response of a three-terminal Carlin circulator to account for transport via a quantum Hall edge (see the Appendix). This yields an expression for the nonreciprocal admittance matrix of the edge, $Y_{\text {edge }}$, as was done in Ref. [9]. Extending the model in Ref. [9], we add an additional admittance term $Y_{p}$ to account for a direct parasitic coupling $C_{p}$ between terminals [see Fig. 2(c)]. We further include the possibility of dissipation $R$, either directly in the chiral EMP mode or elsewhere in the circuit. Given an admittance of the edge state $Y_{\text {edge }}$, the total admittance is then given by

$$
Y_{\text {total }}=\left(I+R Y_{\text {edge }}\right)^{-1} Y_{\text {edge }}+Y_{p},
$$

where $I$ is the identity matrix and where

$$
Y_{\mathrm{p}}=\left(\begin{array}{ccc}
2 c & -c & -c \\
-c & 2 c & -c \\
-c & -c & 2 c
\end{array}\right),
$$

with $c=i \omega C_{p}$, and $\omega$ is the angular frequency of the microwaves. Microwave $S$ parameters can then be calculated as a function of $\omega$ for a given characteristic impedance of the input port $\left(Z_{0}\right)$.

This model qualitatively captures the mechanism of circulation as arising from the interference of the parasitic 
and quantum Hall edge paths. Despite its simplicity, we find that it also accounts for many of the features seen in the experimental data, including forward and reverse circulation, which occurs near the fundamental and first harmonic of the EMP mode, as shown in Figs. 4(c) and 4(d). For features that occur at fields corresponding to integer filling, we find good agreement with the data for parameter values that are consistent with the device geometry and independent transport measurements (see Fig. 4 caption for details). At magnetic fields slightly away from integer filling, increasing $R$ in the model yields similar results to the observed phenomena.

\section{TUNABLE NONRECIPROCITY}

Finally, having outlined the mechanism leading to nonreciprocity in our device, we describe a new mode that has no analog in the operation of classical circulators but may enable reconfigurable passive routing of microwave signals on chip using gate voltages to modulate the velocity of

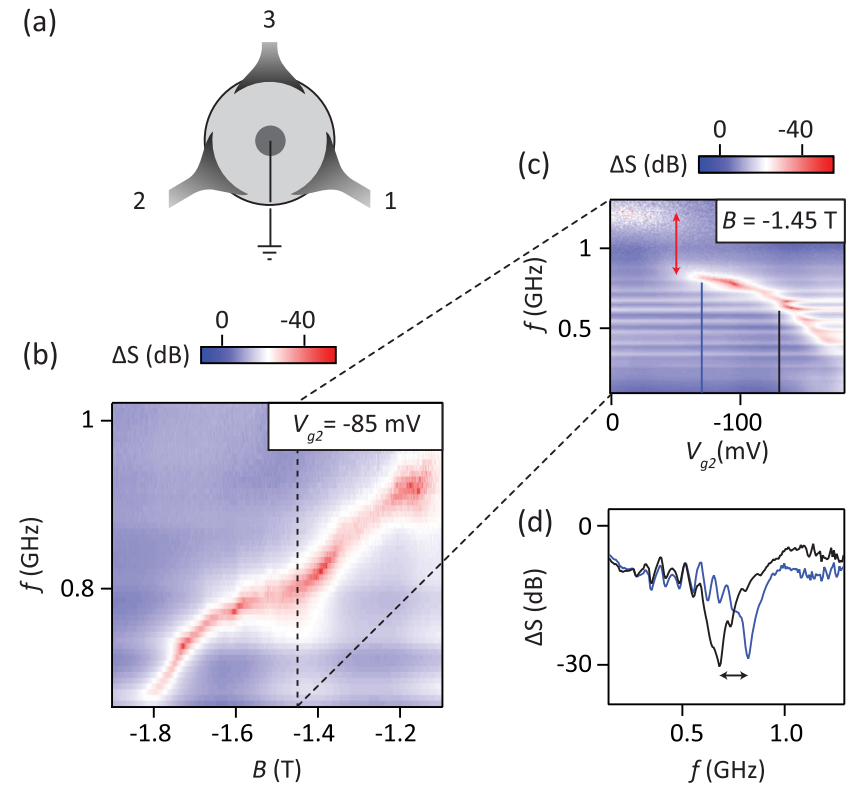

FIG. 5. (a) Schematic of the gate-tunable device, with ports overlapping the edge of the disk and a grounded contact on the mesa. Bias tees enable the application of both rf and dc voltages. A large nonreciprocity $\Delta S=S_{13}-S_{23}$ is observed as a function of magnetic field, as shown in (b) for the case $V_{g 2}=-85 \mathrm{mV}$. At fixed negative magnetic field values, varying $V_{g 2}$ is found to affect the path from ports 3 to 1 , and this produces a significant modulation in the frequency response of the circulator [shown in (c) and (d)]. This response is mirrored with a change of sign $\Delta S$ at positive magnetic field values, where the direction of EMP propagation is reversed and $V_{g 1}$ is varied (see Ref. [28]). The red arrow in (c) indicates a discontinuous jump in frequency as a gate voltage is first applied, while vertical lines show the positions of 1D cuts presented in (d). Horizontal striations in (c) are the result of small standing waves associated with an impedance mismatch between the amplifier and the device.
EMPs. To demonstrate this mode, we make use of an alternate device [Fig. 5(a)], where, in comparison to the previous device, the port electrodes are positioned to now overlap the edge and a grounded contact is added to the center of the disk. Sweeping the magnetic field, we find this device exhibits regions of strong nonreciprocity, as shown in Fig. 5(b). Tunable nonreciprocity is demonstrated at a fixed negative $B$ field by sweeping the dc voltage applied to the port-2 gate $V_{g 2}$. This adjusts the response between the source and sink ports 3 and 1 , respectively, which tunes the frequency of isolation $\Delta S=S_{13}-S_{23}$ as shown in Figs. 5(c) and 5(d). Applying a voltage to a gate hardly modifies the total path length of the EMP in this geometry, but it can lead to a significant modulation in its velocity by varying the carrier density, electric field, or extent of screening at the disk boundary [25,36]. As a function of $V_{g 2}$, Fig. 5(c) shows that the nonreciprocal response of the circulator initially drops from about $1.2 \mathrm{GHz}$ to about $0.8 \mathrm{GHz}$ as the gate voltage is initially applied, followed by a more gradual decrease in the center frequency as the gate is made increasingly negative. At present, we do not understand why a modest gate voltage leads to a significant velocity modulation and, therefore, frequency response over such a large bandwidth (exceeding $1 \mathrm{GHz}$ and many linewidths in this device). An alternate means of reconfiguring the device can be achieved by adjusting the external magnetic field (as shown in Ref. [28]). In this way, the circulator can produce forward or reverse circulation, selectively routing microwave packets to alternate ports depending on the value of the magnetic field. For such an application, generating the magnetic field on chip using a combination of micromagnets [37] and compact superconducting solenoids could be considered.

\section{CONCLUSION}

We have demonstrated a compact, on-chip microwave circulator based on the nonreciprocal response implicit to the quantum Hall effect. With better matching between the port impedance and impedance of the quantum Hall edge, these highly compact devices can immediately compete with today's commercially available bulky circulators for cryogenic applications. To this end, we draw attention to recent theoretical works [34,35] that suggest new configurations for achieving "self-matching" of the circulator to the characteristic impedance of the ports. Beyond the simple circulator devices demonstrated here, we conclude by noting that an edge state can be considered as a mesoscale delay line with a dynamic, gate-tunable wideband response. Such a dependence opens the prospect of compact, parametric devices such as amplifiers, nonreciprocal filters, and mixers based on the plasmonic and chiral response of the quantum Hall effect. Indeed, such modes can also likely be realized at zero magnetic field using topological insulator devices that exhibit the quantum anomalous Hall effect [38]. 


\section{ACKNOWLEDGMENTS}

We thank D. DiVincenzo, C. Nayak, and J. Cano for useful conversations. This research was supported by Microsoft Research, the US Army Research Office Grant No. W911NF-14-1-0097, and the Australian Research Council Centre of Excellence Scheme (Grant No. EQuS CE110001013).

A. C. M. and J. I. C. contributed equally to this work.

\section{APPENDIX: DETAILS OF MODEL}

We begin with the admittance matrix $Y_{\text {edge }}$ for a threeport quantum Hall circulator using a Carlin-type device, exactly as described in Ref. [9]:

$$
Y_{\text {edge }}(\omega)=\left(\begin{array}{ccc}
i a & b & -b^{*} \\
-b^{*} & i a & b \\
b & -b^{*} & i a
\end{array}\right),
$$

where, as in Ref. [9],

$$
\begin{gathered}
a=\frac{2 \sigma_{\text {edge }} \sin \frac{\omega C_{\text {edge }}}{\sigma_{\text {edge }}}}{1+2 \cos \frac{\omega C_{\text {edge }}}{\sigma_{\text {edge }}}}, \\
b=\sigma_{\text {edge }} \frac{-1+e^{\left(-i \omega C_{\text {edge }} / \sigma_{\text {edge }}\right)}}{1+2 \cos \frac{\omega C_{\text {edge }}}{\sigma_{\text {edge }}}},
\end{gathered}
$$

with $\omega$ the angular frequency, $\sigma_{\text {edge }}$ the conductance of the edge $\left(1 / \sigma_{\text {edge }}=R_{x y}\right)$, and $C_{\text {edge }}$ the contact capacitance between each of the port terminals and the edge. Note that this $3 \times 3$ matrix is not symmetric, so it captures the nonreciprocal response of the chiral edge magnetoplasmons.

We now modify the circuit with respect to Ref. [9], by adding terms to account for dissipation $R$, either along the quantum Hall edge or elsewhere in the circuit, as well as a direct parasitic capacitive path $C_{p}$, between port terminals. We derive an admittance matrix $Y_{\text {total }}$ which captures these additional terms as a function of $Y_{\text {edge }}, R$, and $C_{p}$.

Referring to the circuit diagram shown in Ref. [28], Fig. S2, and given Ohm's law, we write

$$
\vec{I}^{\prime}=Y_{\text {edge }} \vec{V}^{\prime} \quad \text { and } \quad \vec{V}=\vec{V}^{\prime}+R \vec{I}^{\prime},
$$

where $\vec{I}^{\prime}\left(\vec{V}^{\prime}\right)$ are the vectors of currents (voltages) of $Y_{\text {edge }}$ and $\vec{I}(\vec{V})$ are the vectors of currents (voltages) of $Y_{\text {total }}$. Rearranging and substituting, we find

$$
\vec{I}^{\prime}=Y_{\text {res }} \vec{V}
$$

where

$$
Y_{\text {res }}=\left(\mathrm{I}+R Y_{\text {edge }}\right)^{-1} Y_{\text {edge }} \vec{V}
$$

and $I$ is the identity matrix.

We can then write out the individual components of $\vec{I}$ by summing the net flows in and out of each port of the circulator:

$$
\begin{aligned}
& I_{1}=I^{\prime}{ }_{1}+\left(I_{12}-I_{31}\right)=\left(Y_{\text {res }}^{11} V_{1}+Y_{\text {res }}^{12} V_{2}+Y_{\text {res }}^{13} V_{3}\right)+i \omega C_{p}\left(2 V_{1}-V_{2}-V_{3}\right), \\
& I_{2}=I^{\prime}{ }_{2}+\left(I_{23}-I_{12}\right)=\left(Y_{\text {res }}^{21} V_{1}+Y_{\text {res }}^{22} V_{2}+Y_{\text {res }}^{23} V_{3}\right)+i \omega C_{p}\left(2 V_{2}-V_{3}-V_{1}\right), \\
& I_{3}=I^{\prime}{ }_{3}+\left(I_{31}-I_{23}\right)=\left(Y_{\text {res }}^{31} V_{1}+Y_{\text {res }}^{32} V_{2}+Y_{\text {res }}^{33} V_{3}\right)+i \omega C_{p}\left(2 V_{3}-V_{1}-V_{2}\right)
\end{aligned}
$$

and thus,

$$
\vec{I}=Y_{\text {res }} \vec{V}+\left(\begin{array}{ccc}
2 i \omega C_{p} & -i \omega C_{p} & -i \omega C_{p} \\
-i \omega C_{p} & 2 i \omega C_{p} & -i \omega C_{p} \\
-i \omega C_{p} & -i \omega C_{p} & 2 i \omega C_{p}
\end{array}\right) \vec{V}
$$

such that we can write

$$
\vec{I}=Y_{\text {total }} \vec{V},
$$

where

$$
\begin{aligned}
Y_{\text {total }}= & \left(\mathrm{I}+R Y_{\text {edge }}\right)^{-1} Y_{\text {edge }} \\
& +\left(\begin{array}{ccc}
2 i \omega C_{p} & -i \omega C_{p} & -i \omega C_{p} \\
-i \omega C_{p} & 2 i \omega C_{p} & -i \omega C_{p} \\
-i \omega C_{p} & -i \omega C_{p} & 2 i \omega C_{p}
\end{array}\right)
\end{aligned}
$$

Finally, we calculate the scattering parameters of the system as a function of the angular frequency $\omega$ via the transformation

$$
S(\omega)=\left(Z^{-1}+Y_{\text {total }}\right)^{-1}\left(Z^{-1}-Y_{\text {total }}\right),
$$

where $Z=Z_{0} \mathrm{I}$. This expression is used to calculate $S_{31}$ and $S_{13}$ and their difference, as shown in Fig. 4(d). To compare our model to experimental data in Fig. 4(c), where the background response at zero magnetic field has been subtracted, we normalize the results of the model by setting to $S=0 \mathrm{~dB}$ the response via just the parasitic path $C_{p}$. Thus, a value of $S=0 \mathrm{~dB}$ in Figs. 4(c) and 4(d) represents the response of the system when no quantum Hall edge is present, similar to the experimental situation at zero field. 
To qualitatively compare our model to the experimental data, we account for the five variables: the characteristic impedance $Z_{0}$, the parasitic capacitance $C_{p}$, the effective capacitance of the quantum Hall edge $C_{\text {edge }}$, the conductance of the edge $\sigma_{\text {edge }}=1 / R_{x y}$, and the total dissipation $R$. To produce qualitative fits to the data, we fix $Z_{0}$ and vary $R_{x y}$ and $C_{\text {edge }}$ to align the peaks and dips to correspond to the EMP frequencies. Finally, we adjust $C_{p}$ and $R$ to vary the amplitude and width of the features in our model to fit the corresponding data. Although qualitative, the values of $C_{p}$ and $R$ chosen in the model are consistent with typical values for the parasitic capacitance and total dissipation estimated via transport measurements and geometric considerations.

[1] R. Fleury, D. L. Sounas, C. F. Sieck, M. R. Haberman, and A. Alù, Sound Isolation and Giant Linear Nonreciprocity in a Compact Acoustic Circulator, Science 343, 516 (2014).

[2] L. Feng, M. Ayache, J. Huang, Y. L. Xu, M. H. Lu, Y. F. Chen, Y. Fainman, and A. Scherer, Nonreciprocal Light Propagation in a Silicon Photonic Circuit, Science 333, 729 (2011).

[3] L. Bi, J. Hu, P. Jiang, D. H. Kim, G. F. Dionne, L. C. Kimerling, and C. A. Ross, On-Chip Optical Isolation in Monolithically Integrated Non-Reciprocal Optical Resonators, Nat. Photonics 5, 758 (2011).

[4] N. A. Estep, D. L. Sounas, J. Soric, and A. Alù, MagneticFree Non-Reciprocity and Isolation Based on Parametrically Modulated Coupled-Resonator Loops, Nat. Phys. 10, 923 (2014).

[5] T. M. Stace, C. H. W. Barnes, and G. J. Milburn, Mesoscopic One-Way Channels for Quantum State Transfer via the Quantum Hall Effect, Phys. Rev. Lett. 93, 126804 (2004).

[6] J. Kerckhoff, K. Lalumière, B. J. Chapman, A. Blais, and K. W. Lehnert, On-Chip Superconducting Microwave Circulator from Synthetic Rotation, Phys. Rev. Applied 4, 034002 (2015).

[7] B. Abdo, K. Sliwa, L. Frunzio, and M. Devoret, Directional Amplification with a Josephson Circuit, Phys. Rev. X 3, 031001 (2013).

[8] K. M. Sliwa, M. Hatridge, A. Narla, S. Shankar, L. Frunzio, R. J. Schoelkopf, and M. H. Devoret, Reconfigurable Josephson Circulator/Directional Amplifier, Phys. Rev. X 5, 041020 (2015).

[9] G. Viola and D. P. DiVincenzo, Hall Effect Gyrators and Circulators, Phys. Rev. X 4, 021019 (2014).

[10] W. P. Mason, W. H. Hewitt, and R. F. Wick, Hall Effect Modulators and "Gyrators" Employing Magnetic Field Independent Orientations in Germanium, J. Appl. Phys. 24, 166 (1953).

[11] R. F. Wick, Solution of the Field Problem of the Germanium Gyrator, J. Appl. Phys. 25, 741 (1954).

[12] M. Büttiker, Absence of Backscattering in the Quantum Hall Effect in Multiprobe Conductors, Phys. Rev. B 38, 9375 (1988).
[13] D. J. Reilly, C. M. Marcus, M. P. Hanson, and A. C. Gossard, Fast Single-Charge Sensing with a rf Quantum Point Contact, Appl. Phys. Lett. 91, 162101 (2007).

[14] C. Barthel, D. J. Reilly, C. M. Marcus, M. P. Hanson, and A. C. Gossard, Rapid Single-Shot Measurement of a SingletTriplet Qubit, Phys. Rev. Lett. 103, 160503 (2009).

[15] J. I. Colless, A. C. Mahoney, J. M. Hornibrook, A. C. Doherty, H. Lu, A. C. Gossard, and D. J. Reilly, Dispersive Readout of a Few-Electron Double Quantum Dot with Fast rf Gate Sensors, Phys. Rev. Lett. 110, 046805 (2013).

[16] J. J. Pla, K. Y. Tan, J. P. Dehollain, W. H. Lim, J. J. L. Morton, D. N. Jamieson, A. S. Dzurak, and A. Morello, A Single-Atom Electron Spin Qubit in Silicon, Nature 489, 541 (2012).

[17] A. E. Miroshnichenko, S. Flach, and Y. S. Kivshar, Fano Resonances in Nanoscale Structures, Rev. Mod. Phys. 82, 2257 (2010).

[18] D. B. Mast, A. J. Dahm, and A. L. Fetter, Observation of Bulk and Edge Magnetoplasmons in a Two-Dimensional Electron Fluid, Phys. Rev. Lett. 54, 1706 (1985).

[19] D. C. Glattli, E. Y. Andrei, G. Deville, J. Poitrenaud, and F. I. B. Williams, Dynamical Hall Effect in a TwoDimensional Classical Plasma, Phys. Rev. Lett. 54, 1710 (1985).

[20] V. A. Volkov and S. A. Mikhailov, Edge Magnetoplasmons: Low Frequency Weakly Damped Excitations in Homogeneous Two-Dimensional Electron Systems, Zh. Eksp. Teor. Fiz. 94, 217 (1988) [Sov. Phys. JETP 67, 1639 (1988)].

[21] E. Y. Andrei, D. C. Glattli, F. I. B. Williams, and M. Heiblum, Low Frequency Collective Excitations in the Quantum-Hall System, Surf. Sci. 196, 501 (1988).

[22] V. K. Talyanskii, M. Wassermeier, A. Wixforth, J. Oshinowo, J. P. Kotthaus, I. E. Batov, G. Weimann, H. Nickel, and W. Schlapp, Edge Magnetoplasmons in the Quantum Hall Effect Regime, Surf. Sci. 229, 40 (1990).

[23] R. C. Ashoori, H. L. Stormer, L. N. Pfeiffer, K. W. Baldwin, and K. West, Edge Magnetoplasmons in the Time Domain, Phys. Rev. B 45, 3894 (1992).

[24] N. B. Zhitenev, R. J. Haug, K. v Klitzing, and K. Eberl, Experimental Determination of the Dispersion of Edge Magnetoplasmons Confined in Edge Channels, Phys. Rev. B 49, 7809 (1994).

[25] N. Kumada, S. Tanabe, H. Hibino, H. Kamata, M. Hashisaka, K. Muraki, and T. Fujisawa, Plasmon Transport in Graphene Investigated by Time-Resolved Electrical Measurements, Nat. Commun. 4, 1363 (2013).

[26] I. Petković, F. I. B. Williams, K. Bennaceur, F. Portier, P. Roche, and D. C. Glattli, Carrier Drift Velocity and Edge Magnetoplasmons in Graphene, Phys. Rev. Lett. 110, 016801 (2013).

[27] N. Kumada, P. Roulleau, B. Roche, M. Hashisaka, H. Hibino, I. Petković, and D.C. Glattli, Resonant Edge Magnetoplasmons and Their Decay in Graphene, Phys. Rev. Lett. 113, 266601 (2014).

[28] See Supplemental Material at http://link.aps.org/ supplemental/10.1103/PhysRevX.7.011007 for further discussion, and details of experimental methods. 
[29] N. Kumada, H. Kamata, and T. Fujisawa, Edge Magnetoplasmon Transport in Gated and Ungated Quantum Hall Systems, Phys. Rev. B 84, 045314 (2011).

[30] H. Kamata, T. Ota, K. Muraki, and T. Fujisawa, VoltageControlled Group Velocity of Edge Magnetoplasmon in the Quantum Hall Regime, Phys. Rev. B 81, 085329 (2010).

[31] J. Cano, A. C. Doherty, C. Nayak, and D. J. Reilly, Microwave Absorption by a Mesoscopic Quantum Hall Droplet, Phys. Rev. B 88, 165305 (2013).

[32] N. Q. Balaban, U. Meirav, H. Shtrikman, and V. Umansky, Observation of the Logarithmic Dispersion of HighFrequency Edge Excitations, Phys. Rev. B 55, R13397 (1997).

[33] D. P. Druist, P. J. Turley, K. D. Maranowski, E. G. Gwinn, and A. C. Gossard, Observation of Chiral Surface States in the Integer Quantum Hall Effect, Phys. Rev. Lett. 80, 365 (1998).
[34] S. Bosco, F. Haupt, and D. P. DiVincenzo, Self Impedance Matched Hall-Effect Gyrators and Circulators, arXiv: 1609.06543 (to be published).

[35] B. Placke, S. Bosco, and D. P. DiVincenzo, A Model Study of Present-Day Hall-Effect Circulators, arXiv:1609.09624.

[36] H. Kamata, N. Kumada, M. Hashisaka, K. Muraki, and T. Fujisawa, Fractionalized Wave Packets from an Artificial Tomonaga-Luttinger Liquid, Nat. Nanotechnol. 9, 177 (2014).

[37] M. Pioro-Ladriere, T. Obata, Y. Tokura, Y-S. Shin, T. Kubo, K. Yoshida, T. Taniyama, and S. Tarucha, Electrically Driven Single-Electron Spin Resonance in a Slanting Zeeman Field, Nat. Phys. 4, 776 (2008).

[38] C. Z. Chang, J. Zhang, X. Feng, J. Shen, Z. Zhang, M. Guo, K. Li, Y. Ou, P. Wei, L. L. Wang, and Z. Q. Ji, Experimental Observation of the Quantum Anomalous Hall Effect in a Magnetic Topological Insulator, Science 340, 167 (2013). 